\title{
Simultaneous right- and left-hand adaptation in opposite lateral directions following bidirectional optical displacement
}

\author{
LYDIA M. MARTIN and COLIN V. NEWMAN \\ University of Birmingham, Birmingham B15 2TT, England
}

\begin{abstract}
Perceptual adaptation was investigated under conditions in which 40 subjects successively watched movements of their left and right hands through different prisms that displaced vision of both their hands in opposite lateral directions. Significant adaptation to the prisms was found to occur in opposite directions simultaneously for the same subjects' left and right hands. Results confirm that proprioceptive or sensorimotor changes rather than visual processing changes account for prismatic adaptation under these conditions and illustrate further the dominance of visual over proprioceptive information for object location in conditions of conflict.
\end{abstract}

Numerous investigations have shown how readily and quickly human beings can adapt to optical transformations created by prisms that displace the visual scene laterally (Harris, 1965). With few exceptions (Mikaelian \& Malatesta, 1974), most previous research has investigated the plasticity and adaptability of perception in situations in which the observer has been confronted with an optical displacement in a single direction during the same testing period. Research has sought to determine just what is learned in such perceptual rearrangement situations. Investigators from the time of Harris (1965) onward (e.g., Hardt, Held, \& Steinbach, 1971) have typically used an experimental paradigm in which, during a training session, the observer views one of his hands through a prism while carrying out some motor task. With the prisms removed, posttraining tests are conducted to determine the extent of adaptation in the seen and unseen hands. Typically, there is no transfer of learning from the observed to the unobserved hand, which has led such workers as Templeton, Howard, and Wilkinson (1974) to agree with Harris that the adaptive shift in pointing with the seen hand does not involve a shift in the apparent visual location of an object but arises as a result of changes in the felt position of the observed arm, although these changes have been differently characterized as being proprioceptive (Harris, 1965) or sensorimotor (Hardt et al., 1971) in nature. The hypothesis that change has occurred in the processing of visual information for target position has been rejected on the evidence of a failure to demonstrate transfer of learning to the unseen hand. Since this failure to reject a null hypothesis might be due to suppression of an actual visual shift (or to an insensitive measure of the potential shift), the present experiment sought to provide more convincing support for the claim that changes in felt limb position account for the adaptive shift in such experimental situations by demonstrating adaptation when it is not possible for a visual shift to occur. That is, we sought confirmation of the hypothesis tested in the present experiment that observers' left and right hands can be simultaneously adapted to visual displacement in opposite lateral directions. Mikaelian and Malatesta (1974) have successfully demonstrated adaptation in this situation, although they point out one possible limitation in their methodology, namely, that separate eyes were used during exposure and test. The present experiment avoided this difficulty when testing the same hypothesis.

A simple hand placing task was also investigated in this experiment to confirm that any adaptive shifts in hand location found in the main target-aiming task also apply to a further, simpler task in which subjects were asked merely to place their hands a given distance apart.

\section{METHOD}

\section{Apparatus}

The apparatus was very like that first used by Held and Gottlieb (1958) to study adaptation to prism-induced visual displacement. A rectangular, wooden board with a circular, .5-in. diam, monocular viewing hole at its center was mounted horizontally 12 in. above a table. A target figure consisting of four 4-in. black lines intersecting to form a 3-in. square with all lines extending $.5 \mathrm{in}$. beyond the corners was drawn on a white sheet of paper that was mounted vertically on a board at right angles to the table and viewing hole. This target figure was observed via its reflection in a removable mirror placed obliquely at $45 \mathrm{deg}$ beneath the viewing board and above the table. On the table, in a fixed position under the mirror, were placed squared sheets of paper on which subjects were to draw small dots to record their judgments of the apparent positions of the corners of the target square. With the mirror in place, subjects could not see their own hands or the squared sheets of paper on which they were to record their judgments. Beneath the viewing hole were mounted brackets that permitted either a base-left or a 
base-right 20 -diopter prism to be held immediately beneath the viewing hole, thereby enabling vision to be displaced to right or left or kept undisplaced.

\section{Procedure}

Twenty male and 20 female subjects, none of whom had any clinically diagnosed optical defects requiring the wearing of spectacles, were assigned at random to the counterbalanced testing conditions of the experiment, except that equal numbers of males and females were tested in each condition. Subjects were all undergraduate students, the majority of whom were studying physical education, and all were naive as to the purpose of the experiment. They were tested individually using their preferred eye throughout the experiment, and each underwent the following testing and training phases of the experiment.

Target displacement pretest. With no prism under the viewing hole and the mirror in its oblique position, subjects were asked to draw a dot on the scoring paper fixed to the desk to mark the apparent location of each corner of the target square. Judgments were made in a clockwise direction starting from the top lefthand corner of the square and continuing around the square 10 times. The scoring paper was then changed and the test repeated, so that half of the subjects made judgments first using their right hands and then their left and the other half of the subjects made judgments with their left and then their right hands. The exact positions on the score sheet that were equivalent to the corners of the stimulus square being reflected to a subject's eye had been calculated in advance. The distance each dot deviated to the left or right of a line running vertically through each true target position was recorded as a positive (left) or a negative (right) error. Vertical deviations were ignored. For judgments with each hand, a mean deviation score was later calculated from all $\mathbf{4 0}$ dot placings. This score served as a baseline against which to measure the extent of subsequent prism-induced displacements.

Hand placing pretest. With their hands still obscured by the mirror, subjects were asked to place their index fingers $1 \mathrm{ft}$ apart on the table in front of them. The experimenter measured this distance, which served as a baseline against which to compare later prism-induced changes in apparent distance between the hands. No knowledge of results was given to subjects at any stage in the experiment.

Training session. Either the base-left or the base-right prism was attached beneath the viewing hole and the mirror was removed, permitting subjects to see their hands, but not the target square. With the right-displacing prism in place, half the subjects who had made pretest judgments initially with their right hands were asked to move their right hands back and forth beneath the viewing hole while watching the resulting selfgenerated movement of their own hands through the prism for $1 \mathrm{~min}$. Rate of movement was voluntary, and subjects were not aiming at any specific targets or given feedback on their performance by the experimenter. The subject then withdrew his hand, and the left-displacing prism was placed under the viewing hole to replace the right-displacing one. The subject was then required to view his left hand as he moved it back and forth for a further $1 \mathrm{~min}$ beneath the viewing hole. The prisms were then exchanged again, and the subject was required to view his right hand for 1 min longer via the right-displacing prism. In all, six alternating 1-min training trials were carried out, three with each hand. Conditions for testing subjects were counterbalanced, so that the remaining half of the subjects who had made pretest judgments initially with their right hands were subsequently required to start the training session by viewing their left hands under the right-displacing prism and then their right hands under the leftdisplacing prism. Similarly, the remaining half of the subjects who had made pretest judgments initially with their left hands were divided evenly into the above two training conditions.

Target displacement posttests. As soon as the $6 \mathrm{~min}$ of training had been completed, the prisms were removed from beneath the viewing hole, the oblique mirror was replaced, and subjects completed a second set of judgments in which they indicated the positions of the corners of the target square in precisely the same way as in the target displacement pretests. The subject made the posttest judgment in the same order as in the pretest.

Hand placing posttest. Finally, the subject was asked to repeat the hand placing judgment made in the pretest by placing his index fingers $1 \mathrm{ft}$ apart while they were hidden from sight under the mirror.

\section{RESULTS}

\section{Hand Placing Tests}

Subjects could be divided into two groups: (1) those for whom the predicted adaptive change in apparent location of hand position should be toward the midline of the body, and (2) those for whom the predicted adaptive shift should be away from the midline of the body. In Group 1, the average hand placing pretest judgment of a 1 -ft length was 12.01 in. and the average hand placing posttest judgment was 10.94 in. a significant change of $1.07 \mathrm{in}$. in the predicted direction $[t(19)=3.96, p<.01]$. In Group 2, the average hand placing pretest judgment was 11.36 in. and the average hand placing posttest judgment was 12.03 in., a significant change in the predicted direction of .67 in. [ $\mathrm{t}(19)$ $=2.14, \mathrm{p}<.05]$. When an increase in a subject's pretestto-posttest hand placing scores was recorded as positive and a decrease as negative, the mean Group 1 score of $-1.07 \mathrm{in}$. was significantly different from the mean Group 2 score of +.67 in. $[\mathrm{t}(39)=1.45, \mathrm{p}<.001]$. The apparent difference in the absolute extent of the change in pretest-to-posttest hand placing scores between Groups 1 and 2 (i.e., disregarding direction of the change) was not statistically significant $[t(39)=1.29$, $\mathrm{p}>.05]$. There were no significant differences between the groups in the initial pretest hand placing scores and no differences between the sexes in performance.

\section{Target Displacement Tests}

The order of making judgments in the pre- and posttests using first either the left or the right hand had no significant effect on performance within any condition, and no difference between the sexes was found. Data were thus collapsed across the variables of order and sex to give two experimental groups, 1 and 2 . In Group 1 , it was predicted that the effect of viewing the left hand through the left-displacing prism would result in its apparent location being displaced to the left, giving an erroneous shift to the right from the pre- to posttest judgement. Results showed a pretest mean judgment of .02 in. to the right of the target and a mean posttest judgment. Results showed a pretest mean judgment of $.02 \mathrm{in}$. to the right of the target and a mean posttest $[t(19)=3.55, p<.01]$. Similarly, for the right hand, which was viewed through a right-displacing prism, an erroneous shift to the left was predicted from the pretest to the posttest judgments. Results showed a pretest mean judgment of 22 in. to the right of the target and a mean posttest judgment of .51 in. to the left of the target, a significant shift in the predicted direction of 
.73 in. $[\mathrm{t}(19)=3.76, \mathrm{p}<.01]$. In Group 2, in which subjects viewed the left hand with the right-displacing prism, the predictions were reversed. Results for mean judgments with the left hand were .68 in. to the right of the target on the pretest and $.55 \mathrm{in}$. to the left of the target on the posttest, a shift in the predicted direction of 1.23 in. $[\mathrm{t}(19)=5.9, \mathrm{p}<.01]$; with the right hand, the mean pretest judgment was .45 in. to the right of the target, and the mean posttest judgment was 1.36 in. further to the right of the target, a significant shift of $.91 \mathrm{in}$. in the predicted direction [ $\mathrm{t}(19)=3.75, \mathrm{p}<.01]$.

\section{DISCUSSION}

The target displacement tests convincingly demonstrate that different reafferent visual experiences when viewing the right and left hands through separate prisms giving optical displacements in opposite lateral directions can simultaneously, in the same subjects, produce a situation in which the left hand undergoes an adaptive shift in the dirction opposite to that of the right hand. It must be concluded that it is the felt position of the limb that has undergone a change during the prism adaptation experience and not the apparent visual position of the target that has altered, since the latter position cannot simultaneously exist in two places at once within the same person's perceptual experience. A single viewed point in space must have a unique location, even though a distorted sense of felt limb position may result in errors being made in opposite directions when subjects aim to reach for this point but have hands whose felt positions are distorted in opposite directions simultaneously. The hand placing tests have less important implications, showing only that the prism-induced distortions studied in this experiment are sufficiently all embracing to reveal themselves powerfully even on a very simple motor task that is intrinsically less experimentally contrived than the principal test in this experiment. While essentially confirming, albeit more powerfully, earlier findings on the role of proprioceptive or sensorimotor factors in prism adaptations, these results emphasize how the proprioceptive information on an object's position can be discrepant from limb to limb at the same moment in time.

\section{REFERENCES}

Hardt, M. E., Held, R., \& Steinbach, M. J. Adaptation to displaced vision: A change in the central control of sensorimotor coordination. Journal of Experimental Psychology, 1971, 89, 229-239.

Harris, C. S. Perceptual adaptations to inverted, reversed, and displaced vision. Psychological Review, 1965, 72, 419-444.

Held, R., \& Gottlie B, N. Technique for studying adaptation to disarranged hand-eye coordination. Perceptual and Motor Skills, $1958,8,83-86$.

Mikaelian, H. H., \& Malatesta, V. Specialized adaptation to displaced vision. Perception, 1974, 3, 135-139.

Templeton, W. B., Howard, I. P., \& Wilkinson, D. A. Additivity of components of prismatic adaptation. Perception \& Psychophysics, 1974, 15, 249-257.

(Received for publication October 30, 1980.) 